\title{
102. Deutscher Röntgenkongress: Welche Themen erwarten Sie?
}

„Intelligenz vernetzen“ - beim 102. Deutschen Röntgenkongress steht vor allem die interdisziplinäre Vernetzung im Fokus. Von künstlicher Intelligenz über strukturierte Befundung bis hin zur onkologischen Diagnostik bietet der diesjährige Kongress erneut ein abwechslungsreiches Programm. In der untenstehenden Übersicht finden Sie die Programmübersicht für Juni und Juli. Die genauen Daten und Uhrzeiten können Sie auf www.roentgenkongress.de > Programm einsehen. Im Online-Veranstaltungskalender werden zudem laufend neue Themen ergänzt.

\section{JUNI*:}

\begin{tabular}{|c|c|}
\hline 03.06 .2021 & $\begin{array}{l}\text { - Willkommenskultur in der Radiologie - Geht das überhaupt? } \\
\text { - Muskulo II - Impingementsyndrome }\end{array}$ \\
\hline 09.06 .2021 & - MTRA 6: Strahlenschutz und Dosismanagement \\
\hline 10.06.2021 & $\begin{array}{l}\text { - Neuroradiologie I - Entzündungen des Gehirns } \\
\text { - Neuroradiologie - Aktuelles aus der Wissenschaft } \\
\text { - Neuroradiologie II - Blutungen in der zerebralen Bildgebung }\end{array}$ \\
\hline 12.06 .2021 & $\begin{array}{l}\text { - Herzdiagnostik I - Neue Entwicklungen bei MRT und CT } \\
\text { - Herzdiagnostik II - Aktueller Stand in der quantitativen Bildgebung } \\
\text { - Herzdiagnostik III - Myokarditis, Kardiomyopathien und Herztumoren }\end{array}$ \\
\hline 17.06.2021 & $\begin{array}{l}\text { - Forensische Radiologie I - Was Sie schon immer wissen w(s)ollten } \\
\text { - Forensische Radiologie II: Battered Child }\end{array}$ \\
\hline 19.06.2021 & $\begin{array}{l}\text { - RöKo International: MSK: Al and Tumors } \\
\text { - RöKo International: Chest: Al and Pulmonary Imaging }\end{array}$ \\
\hline 23.06.2021 & - MTRA 7: Technologischer Fortschritt \\
\hline 24.06 .2021 & $\begin{array}{l}\text { - Kinderradiologie II - Sport- und Unfallmedizin bei Kindern und Jugendlichen I } \\
\text { - Kinderradiologie Wissenschaft I - Onkologische Studien in der Kinderradiologie } \\
\text { - Kinderradiologie III - Sport- und Unfallmedizin bei Kindern und Jugendlichen II }\end{array}$ \\
\hline 26.06.2021 & $\begin{array}{l}\text { - Kinderradiologie IV - Künstliche Intelligenz und Radiomics in der Kinderradiologie } \\
\text { - Kinderradiologie Wissenschaft II - Aktuelles zur MRT-Bildgebung in der Kinderradiologie } \\
\text { - Kinderradiologie V - Leitlinien - Aktuelles bei Kindern } \\
\text { - Kinderradiologie VI - Pädiatrische Onkologie - Strukturierte Befundung }\end{array}$ \\
\hline 26.06 .2021 & $\begin{array}{l}\text { - Update CT- und MRT-Bildgebung: Schneller und trotzdem besser in der onkologischen Bildgebung } \\
\text { - Radiologie trifft Physiker } \\
\text { - Neues zum Strahlenschutz in der CT } \\
\text { - Sicherheit im MRT: Empfehlungen zu Implantaten und Kontrastmitteln }\end{array}$ \\
\hline 30.06 .2021 & - MTRA 8: Digitale Netzwerke in der Radiologie \\
\hline
\end{tabular}

JULI*:

\begin{tabular}{|l|l|l}
\hline 01.07 .2021 & - Mammadiagnostik-Wissenschaft - Die PET-MRT in der Mammadiagnostik \\
\hline 03.07 .2021 & - Gastro IV - Leber: Diagnostik \\
& - Gastrointestinale/Abdominaldiagnostik-Wissenschaft - Innovative Leber-Bildgebung I und II \\
& - Gastro VI - Leber: Follow-up \\
\hline 08.07 .2021 & - The whole picture: NHL/CLL \\
\hline 09.07 .2021 & - AiW-Curriculum Interventionelle Radiologie \\
& - Physik für den Facharzt: Strahlenschutz bei Interventionen, inkl. Risikoabschätzung für das Personal \\
& - Fit für den Facharzt - Interventionelle Radiologie - I und II \\
\hline 10.07 .2021 & - Fit für den Facharzt - Neuroradiologie \\
& - Fit für den Facharzt - Kopf/Halsdiagnostik \\
& - FFZ Skills Lab - Klinische Skills: Befunden, begutachten und unterrichten \\
& - Fit für den Facharzt - Muskuloskelettale Radiologie
\end{tabular}

* Änderungen vorbehalten 

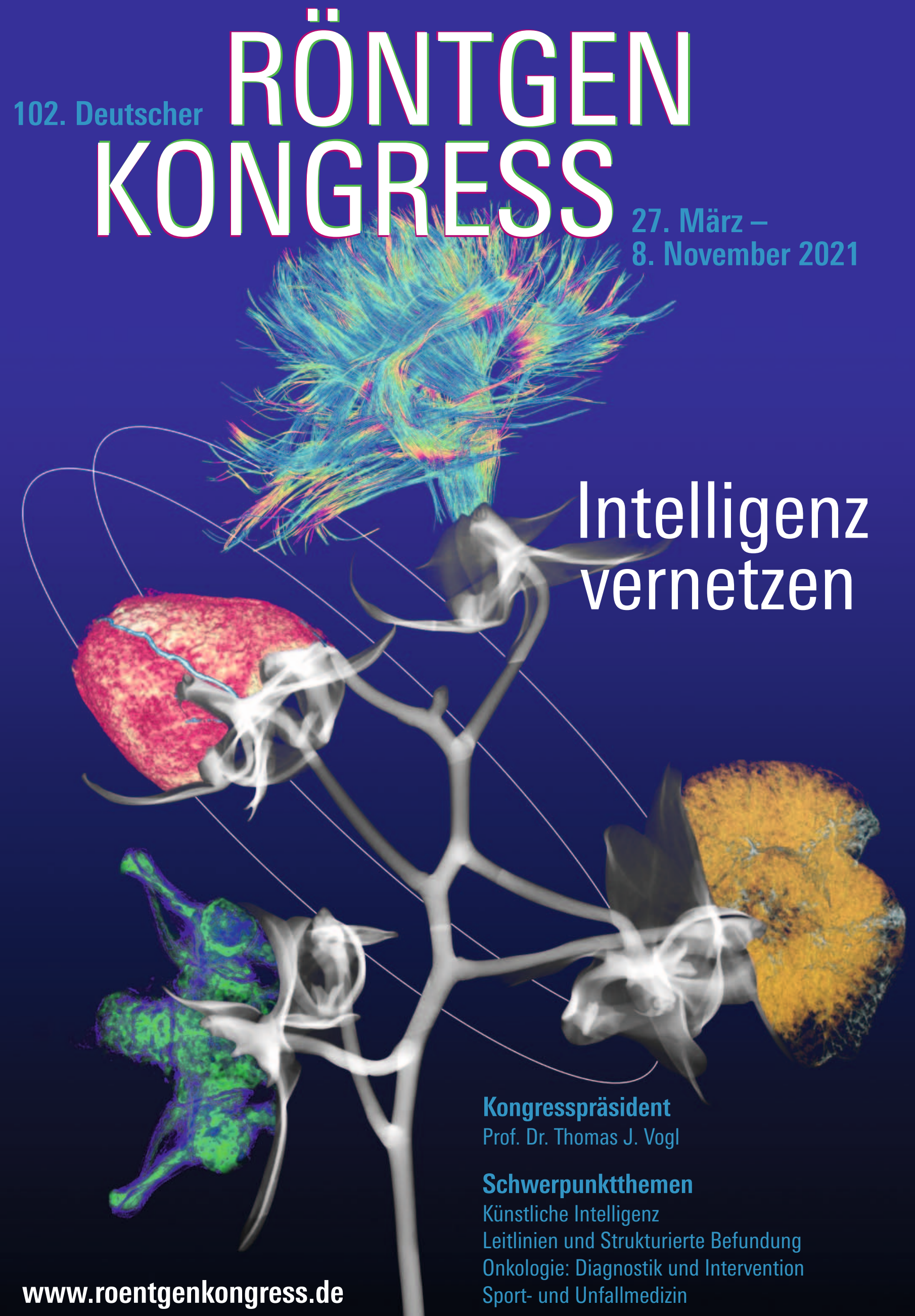


\section{Woche für Woche Fortbildungen und Fachinformationen}

Wie sieht eine typische digitale Röntgenkongress-Woche aus? Hier erhalten Sie einen detaillierten Überblick:

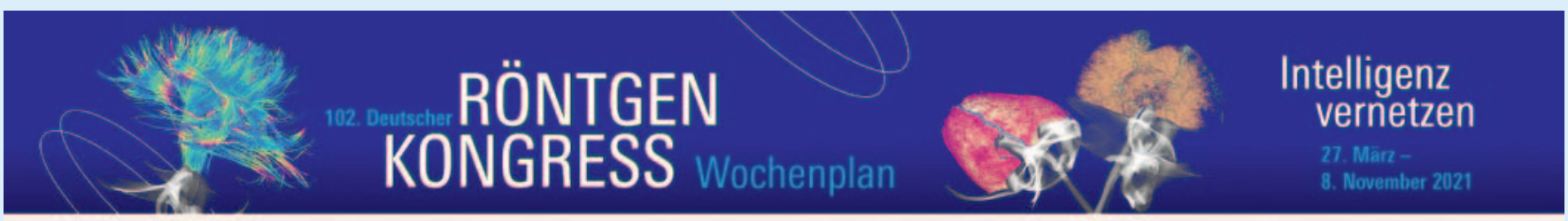

\section{MO} DI MI

DO FR

SA

SO

\section{DEUTSCHER RÖNTGENKONGRESS}

Für DRG-Mitglieder auf conrad: Highlight-Sitzungen, Refresherkurse, „FFF - Fit für den Facharzt", Wissenschaft und MTRA-Fortbildungen On-Demand

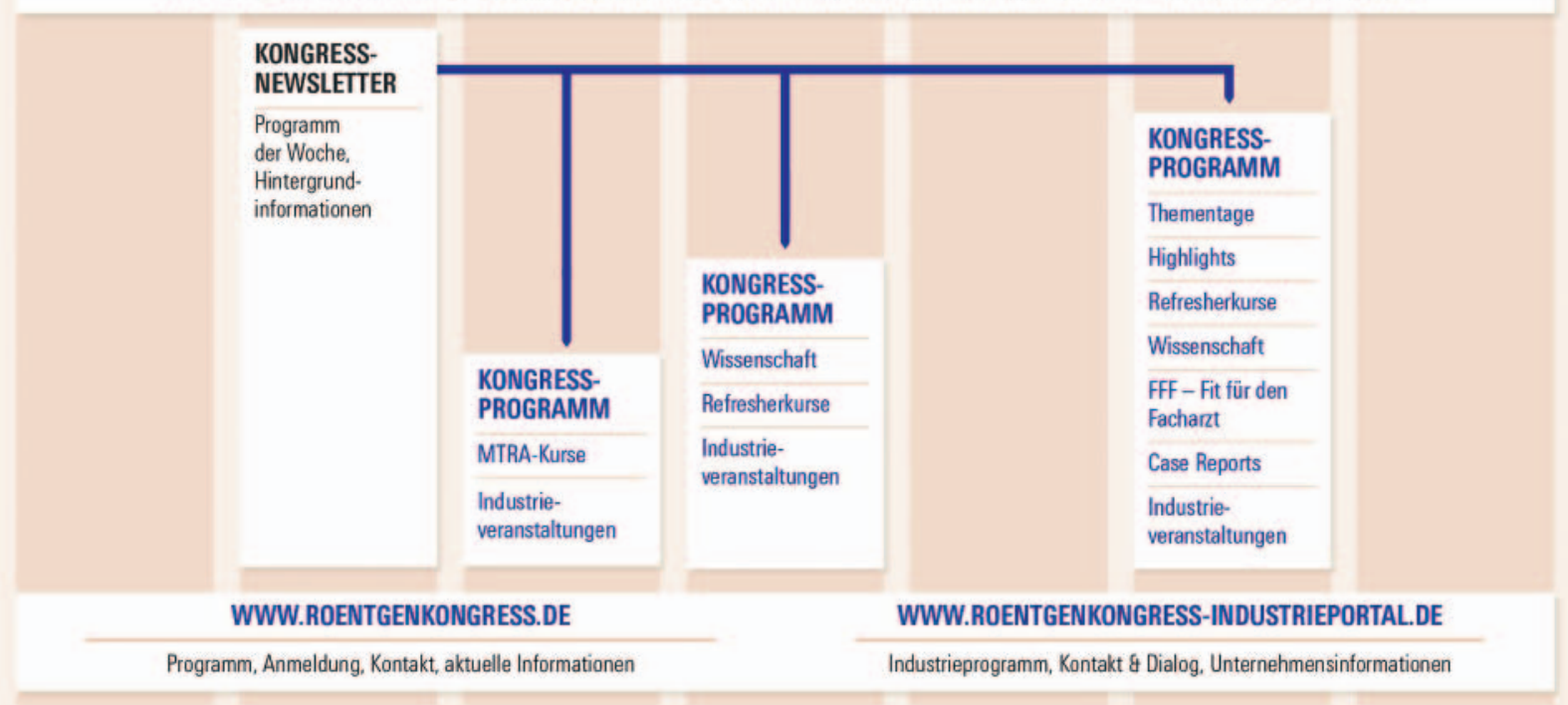

